\title{
Il prelievo selettivo dalle vene surrenaliche nella diagnosi di sottotipo dell'iperaldosteronismo primario
}

\author{
Martina Bollati $^{1} \cdot$ Fabio Bioletto $^{1} \cdot$ Chiara Lopez $^{1} \cdot$ Mirko Parasiliti-Caprino $^{1} \cdot$ Ezio Ghigo $^{1} \cdot$ Mauro Maccario $^{1}$
}

Accettato: 14 giugno 2020 / Pubblicato online: 19 gennaio 2021

(c) The Author(s) 2021

Sommario L'iperaldosteronismo primario è la causa più frequente di ipertensione arteriosa secondaria e si associa ad aumentato rischio cardiovascolare. Il prelievo venoso selettivo surrenalico costituisce il gold standard nella diagnosi di sottotipo tra forme bilaterali e unilaterali, consentendo un adeguato approccio terapeutico. La sua diffusione è limitata in quanto si tratta di un esame di notevole difficoltà tecnica. Pertanto, risulta necessario eseguire tale procedura in centri di riferimento.

Parole chiave Iperaldosteronismo primario - Ipertensione secondaria - Adenoma surrenalico producente aldosterone . Aldosterone · Prelievo venoso selettivo surrenalico

\section{Introduzione}

L'iperaldosteronismo primario (Primary Aldosteronism, PA) rappresenta la forma più frequente di ipertensione arteriosa secondaria: la sua prevalenza supera il $10 \%$ nei soggetti ipertesi inviati in centri specialistici [1] e cresce all'aumentare della severità dell'ipertensione, raggiungendo il 29,1\% nei pazienti con ipertensione arteriosa resistente [2].

Le cause più frequenti di PA sono l'iperplasia surrenalica bilaterale (Idiopathic Adrenal Hyperplasia, IHA) e gli ade-

Proposto da Carla Scaroni.

Informazioni Supplementari La versione online contiene materiale supplementare disponibile su

https://doi.org/10.1007/s40619-021-00815-6.

M. Parasiliti-Caprino

mirko.parasiliticaprino@unito.it

1 S.C. Endocrinologia, Diabetologia e Metabolismo U., Dipartimento di Scienze Mediche, A.O.U. Città della Salute e della Scienza di Torino, Università di Torino, Torino, Italia nomi aldosterone secernenti (Aldosterone Producing Adenoma, APA), che complessivamente rappresentano oltre il $95 \%$ dei casi; cause più rare sono l'iperplasia surrenalica unilaterale (Unilateral Adrenal Hyperplasia, UAH), le forme familiari (Familial Hyperaldosteronism, FH) e i carcinomi cortico-surrenalici (Adrenocortical Carcinoma, ACC) [3]. Una corretta identificazione dei pazienti con PA è fondamentale, dal momento che tale condizione si associa, a parità di fattori di rischio concomitanti, a un' aumentata insorgenza di eventi cardio- e cerebro-vascolari, rispetto all'ipertensione arteriosa essenziale [4]. Inoltre, il trattamento specifico di tale condizione può consentire la regressione del danno d'organo [5] e la riduzione del rischio cardiovascolare [6].

Il work-up diagnostico dei pazienti con PA consta di tre fasi: screening, conferma diagnostica e diagnosi di sottotipo (Fig. 1). Lo screening di PA si basa sulla determinazione della concentrazione di aldosterone plasmatico (PAC) e della renina, espressa come concentrazione diretta (DRC) o come attività reninica plasmatica (PRA) e sul conseguente calcolo del rapporto aldosterone/renina (ARR) [3].

Nei pazienti con ARR positivo si procede al test di conferma, che può essere rappresentato dal test da infusione salina endovenosa, dal test al captopril, dal test al fludrocortisone o dal test da carico salino orale. In accordo con le linee guida (LG) 2016 dell'Endocrine Society (ES) e con le LG 2020 della Società Italiana dell'Ipertensione Arteriosa (SIIA), il test di conferma non risulta necessario nei casi con fenotipo florido di malattia [3, 7].

Nei pazienti in cui sia stata confermata la presenza di PA è necessario, infine, procedere alla diagnosi di sottotipo per distinguere le forme unilaterali dalle forme bilaterali. Una corretta diagnosi di sottotipo è essenziale per un'adeguata gestione terapeutica, poiché l'approccio risulta differente nei due casi: la surrenectomia unilaterale è la terapia di scelta per le forme unilaterali, poiché determina la gua- 
Fig. 1 Work-up diagnostico di PA secondo le LG ES 2016 (da [3]). PA, iperaldosteronismo primario; $L G$, linee guida; $E S$, Endocrine Society; $A R R$, rapporto aldosterone/renina; $P R A$, attività reninica plasmatica; $P A C$, concentrazione di aldosterone plasmatico; $T C$, tomografia computerizzata; $M d C$ : mezzo di contrasto; $A C C$, carcinoma della corteccia surrenalica; $A V S$, prelievo venoso dalle vene surrenaliche; $M R A$, antagonista del recettore dei mineralcorticoidi

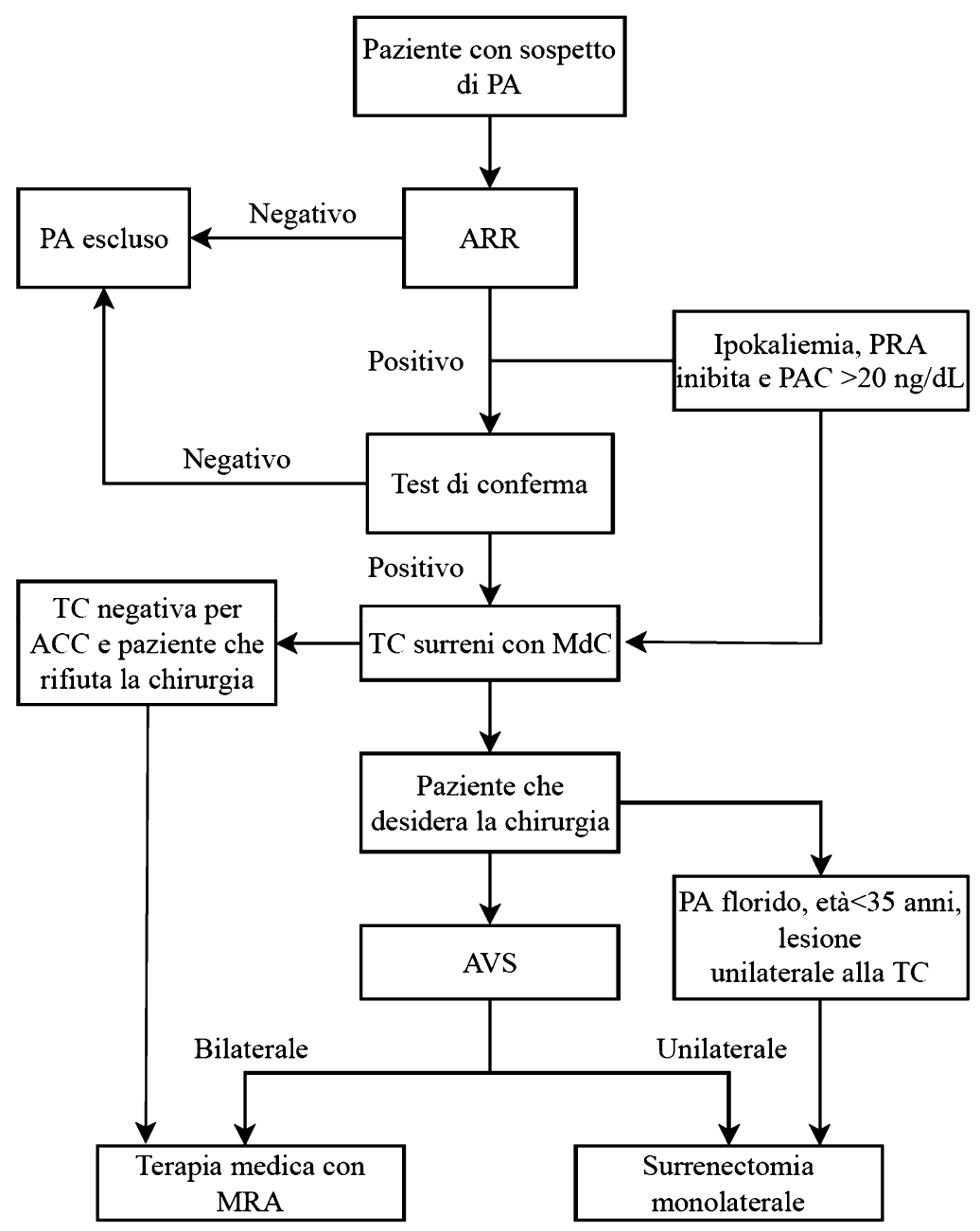

rigione biochimica (intesa come normalizzazione di ARR e kaliemia) in oltre il $90 \%$ dei pazienti e la sospensione o la riduzione del numero dei farmaci anti-ipertensivi in oltre 1' $80 \%$ [8]; nelle forme bilaterali, invece, il trattamento di scelta è la terapia medica con antagonisti del recettore dei mineralcorticoidi (MRA) [3].

Ai fini della diagnosi di sottotipo, in accordo con le LG, è mandatoria l'esecuzione di una TC surrenalica con mezzo di contrasto al fine di valutare la morfologia dei surreni, l'anatomia dei vasi ed escludere l'eventuale presenza di un ACC (Fig. 2). In seconda battuta, nel caso in cui il paziente esprima la volontà di sottoporsi a un eventuale intervento chirurgico, in presenza di ipersecrezione unilaterale di aldosterone, si procede quindi al prelievo selettivo venoso surrenalico (Adrenal Vein Sampling, AVS) per stabilire l'approccio terapeutico più appropriato.

L'AVS rimane infatti, ad oggi, il gold standard di riferimento per la distinzione tra forme di PA unilaterali e bilaterali, risultando più accurato rispetto all'imaging tradizionale $[3,7]$. Tuttavia, la sua diffusione è ancora limitata [9], poiché si tratta di una procedura invasiva e tecnicamente complessa. Inoltre, la mancanza di protocolli standardizzati per la sua esecuzione e interpretazione costituisce un ulteriore elemento di ostacolo alla sua diffusione [10].

\section{Procedura}

\section{Preparazione del paziente}

L'ipokaliemia, presente in circa la metà dei pazienti con APA e in circa il $20 \%$ dei pazienti con IHA [1], deve essere corretta con supplementazione per via orale o endovenosa prima dell'esecuzione della procedura, poiché può inibire la secrezione di aldosterone e, quindi, determinare risultati falsamente negativi.

In previsione dell'esecuzione dell'AVS è inoltre necessario sospendere tutti i farmaci interferenti con il sistema renina-angiotensina-aldosterone; in particolare, $i$ farmaci che aumentano i livelli di renina possono stimolare la secrezione di aldosterone da parte del surrene sano e, quindi, mascherare un'eventuale lateralizzazione [11]. I calcioantagonisti e gli $\alpha_{1}$-bloccanti sono dunque le categorie di 
Fig. 2 Immagine TC di lesioni surrenaliche bilaterali in uomo di 57 anni con successiva evidenza di lateralizzazione destra della secrezione di aldosterone al prelievo venoso selettivo surrenalico

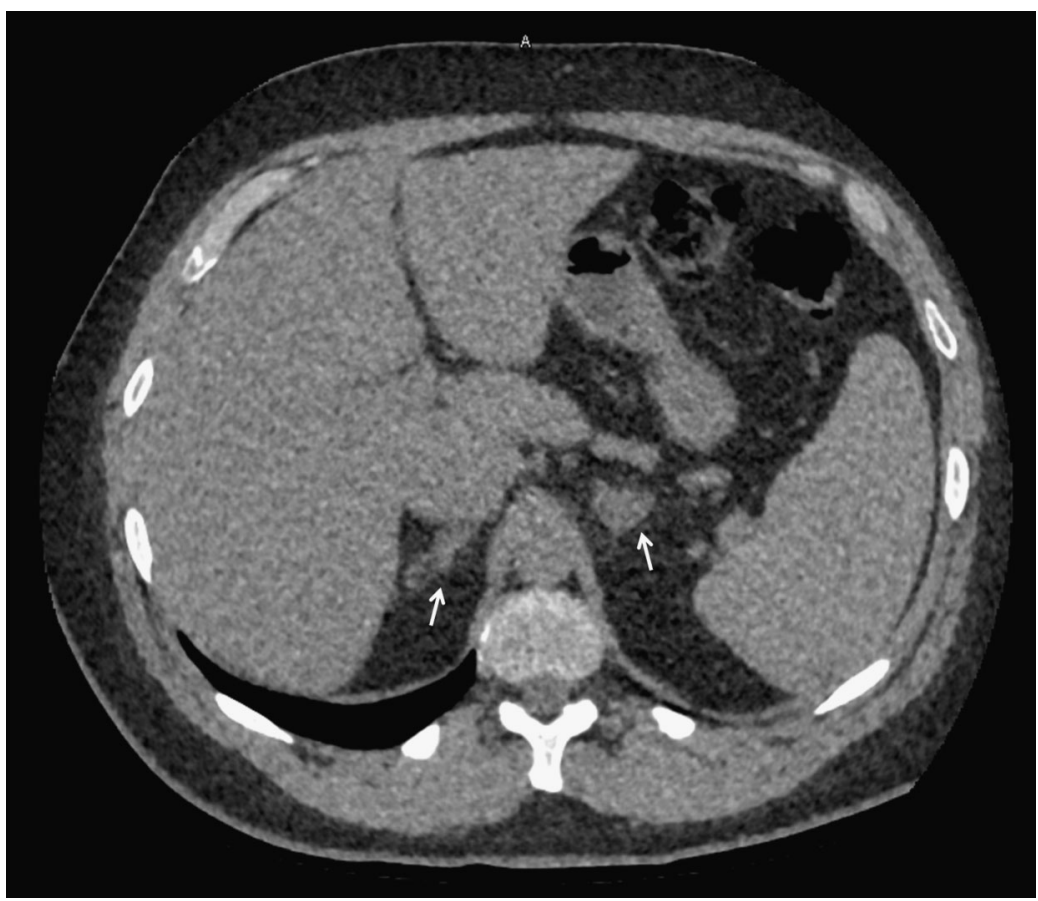

farmaci anti-ipertensivi consigliate nel corso dell'iter diagnostico di PA, poiché non alterano in modo significativo la secrezione di renina. Nei pazienti in cui tali farmaci non siano sufficienti a controllare la pressione arteriosa, può essere consentito l'uso di $\beta$-bloccanti, inibitori dell'enzima di conversione dell' angiotensina (ACE-I) e bloccanti del recettore 1 dell' angiotensina II (ARB), a patto che anche in corso di tali terapie la secrezione di renina risulti soppressa [10, 11]. I diuretici, in particolare gli MRA, dovrebbero essere sospesi almeno 4-6 settimane prima della procedura ma, nei pazienti con ipertensione arteriosa di grado severo o resistente, alcuni autori sostengono che la terapia possa essere mantenuta, purché si verifichi che la renina risulti soppressa $[10,11]$.

\section{Tecnica}

L'AVS prevede la cateterizzazione sotto guida fluoroscopica delle vene surrenaliche attraverso un catetere inserito per via percutanea tramite un accesso venoso femorale (Fig. 3). Una volta verificato il corretto posizionamento del catetere tramite l'iniezione di un piccolo volume di mezzo di contrasto, vengono prelevati dei campioni ematici a livello di entrambe le vene surrenaliche e della vena cava inferiore (VCI) per il dosaggio di cortisolo e aldosterone. L'incannulamento della vena surrenalica sinistra è in genere più semplice e avviene a livello della congiunzione con la vena frenica inferiore, con cui forma un tronco comune che drena nella vena renale sinistra. L'incannulamento della vena surrenalica destra è di solito più complesso, per le sue piccole dimensioni, per il drenaggio ad angolo acuto nella VCI e per la difficoltà nel distinguerla dai piccoli vasi adiacenti, tra cui i rami accessori delle vene epatiche [11].

L'esperienza del radiologo interventista è l'elemento più importante per il successo della procedura, essendo correlata non solo a una maggior percentuale di corretto incannulamento delle vene surrenaliche, ma anche a un tasso più basso di complicanze peri-procedurali [9].

\section{Stimolazione con cosintropina}

L'AVS può prevedere o meno la stimolazione con cosintropina, analogo sintetico dell' ACTH umano corrispondente alla porzione 1-24 dell'ormone. Esistono due protocolli di somministrazione: il primo prevede l'infusione continua alla velocità di $50 \mu \mathrm{g} / \mathrm{h}$ a partire da 30 ' prima e durante la procedura, mentre il secondo prevede la somministrazione di $250 \mu \mathrm{g}$ a bolo [3].

L'obiettivo della stimolazione con cosintropina è quello di massimizzare il gradiente di cortisolo tra le vene surrenaliche e la periferia, minimizzare le fluttuazioni di cortisolo e aldosterone e massimizzare la produzione di aldosterone da parte di un eventuale APA [10]. A tal proposito, nei centri periferici, in cui non vi è la disponibilità di un radiologo interventista esperto nell'esecuzione della procedura, sarebbe preferibile il ricorso alla stimolazione con cosintropina, che si associa a un aumento del tasso di successo dell'incannulamento venoso.

La stimolazione con cosintropina risulta in ogni caso necessaria in alcune specifiche situazioni, ovvero nei pazienti con anamnesi di reazione allergica al mezzo di contrasto iodato che richiedano profilassi con corticosteroidi, nei 
Fig. 3 Immagini radioscopiche di prelievo venoso selettivo dalle vene surrenaliche sinistra (a) e destra (b-d) in una donna di 36 anni con diagnosi di PA e masse surrenaliche bilaterali. I riquadri c, $\mathbf{d}$ mostrano acquisizioni con sottrazione di immagine per favorire una migliore visualizzazione anatomica
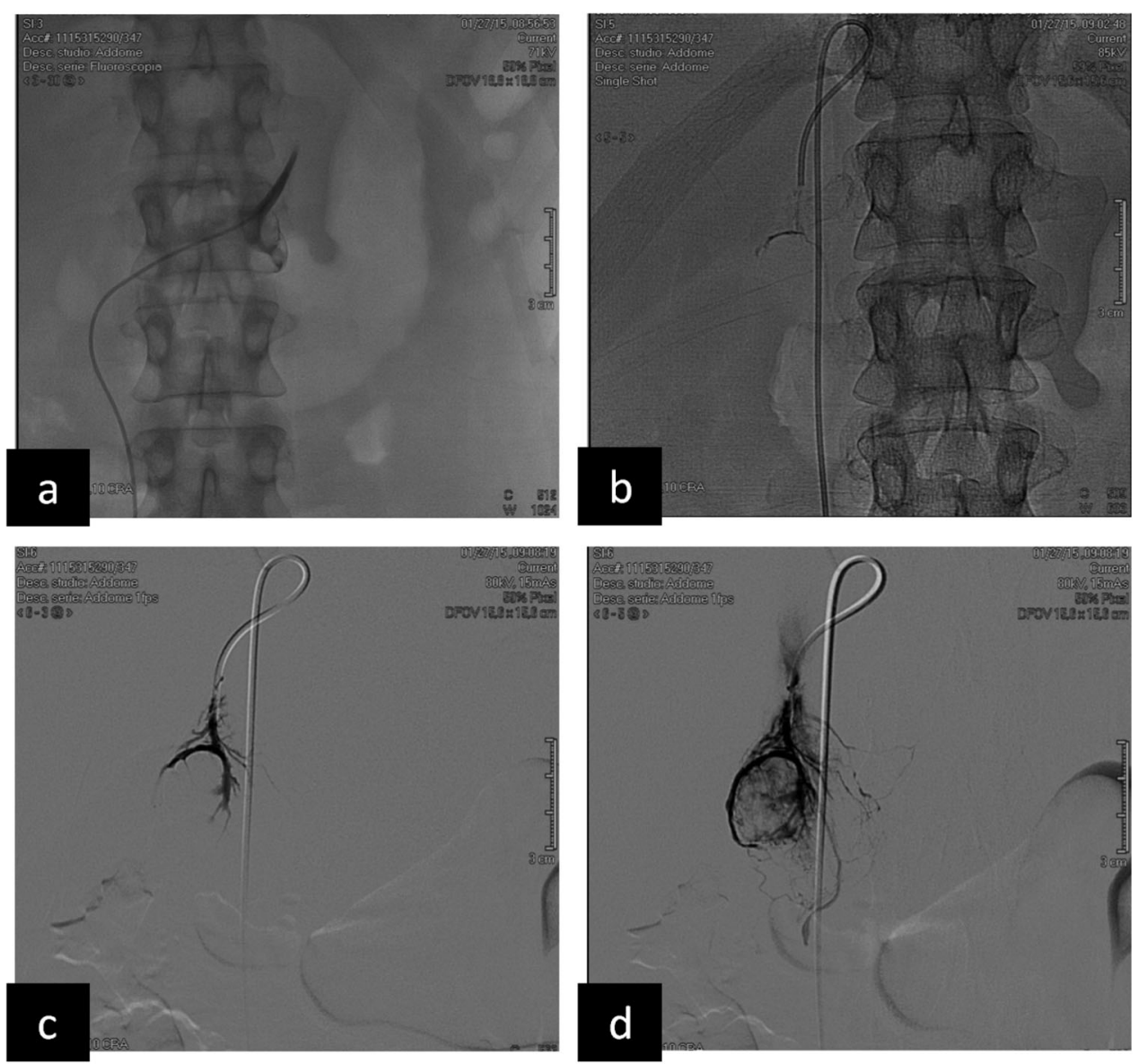

pazienti con concomitante secrezione autonoma di cortisolo (nei quali ci si attende che la secrezione di cortisolo da parte della ghiandola controlaterale possa essere inibita) e qualora l'AVS non venga eseguito al mattino presto (momento in cui la secrezione di ACTH endogeno è massima) [11].

\section{Incannulamento simultaneo e sequenziale}

Nel caso degli AVS non stimolati, alcuni autori hanno suggerito che l'incannulamento simultaneo delle vene surrenaliche possa evitare l'effetto delle fisiologiche oscillazioni nella secrezione di cortisolo e aldosterone, che potrebbero creare dei gradienti artificiali tra le due vene surrenaliche nel caso di un prelievo sequenziale. Tuttavia, a fronte di una maggiore complessità tecnica e di una maggiore invasività (determinata dalla necessità di inserzione contemporanea di due cateteri), non sembrano esistere, ad oggi, dati che confermino la superiorità diagnostica del prelievo simultaneo rispetto a quello sequenziale, in corso di AVS non stimolato [10]. È stato infatti evidenziato come, all'atto pratico, non sussista una significativa discordanza tra i risultati delle due tecniche, purché la distanza temporale tra il prelievo nelle due vene surrenaliche nell' AVS sequenziale sia breve [11].

\section{Dosaggio estemporaneo del cortisolo}

Al fine di aumentare la percentuale di procedure idonee e ottimizzarne l'accuratezza diagnostica, alcuni autori indicano l'uso della determinazione estemporanea del cortisolo nel corso dell'AVS [10, 11]. Abitualmente, i dosaggi ormonali sono disponibili solo dopo il termine della procedura, consentendo un giudizio unicamente retrospettivo sull'idoneità dell'esame. Per ovviare a questo problema, in alcuni centri si utilizza un dosaggio intra-procedurale rapido del cortisolo, che fornisce al radiologo un feedback immediato sull'avvenuta cateterizzazione delle vene surrenaliche, permettendo, in caso di insuccesso, di eseguire ulteriori tentativi di posizionamento del catetere, prima di terminare la procedura [12].

\section{Complicanze}

La complicanza più frequente della procedura è la rottura della vena surrenalica con conseguente formazione di un ematoma intra- o peri-ghiandolare (Fig. 4); sono inoltre stati descritti casi di dissezione e trombosi della vena surrenalica e di infarto surrenalico. La rottura della vena surrenalica è caratterizzata da comparsa di dolore persistente dopo la procedura che richiede elevate dosi di analgesici per 24-48 ore; 
Fig. 4 Immagini radioscopiche progressive di prelievo venoso selettivo da vena surrenalica destra in una donna di 39 anni con evidenza di rottura di un piccolo vaso e spandimento di mezzo di contrasto
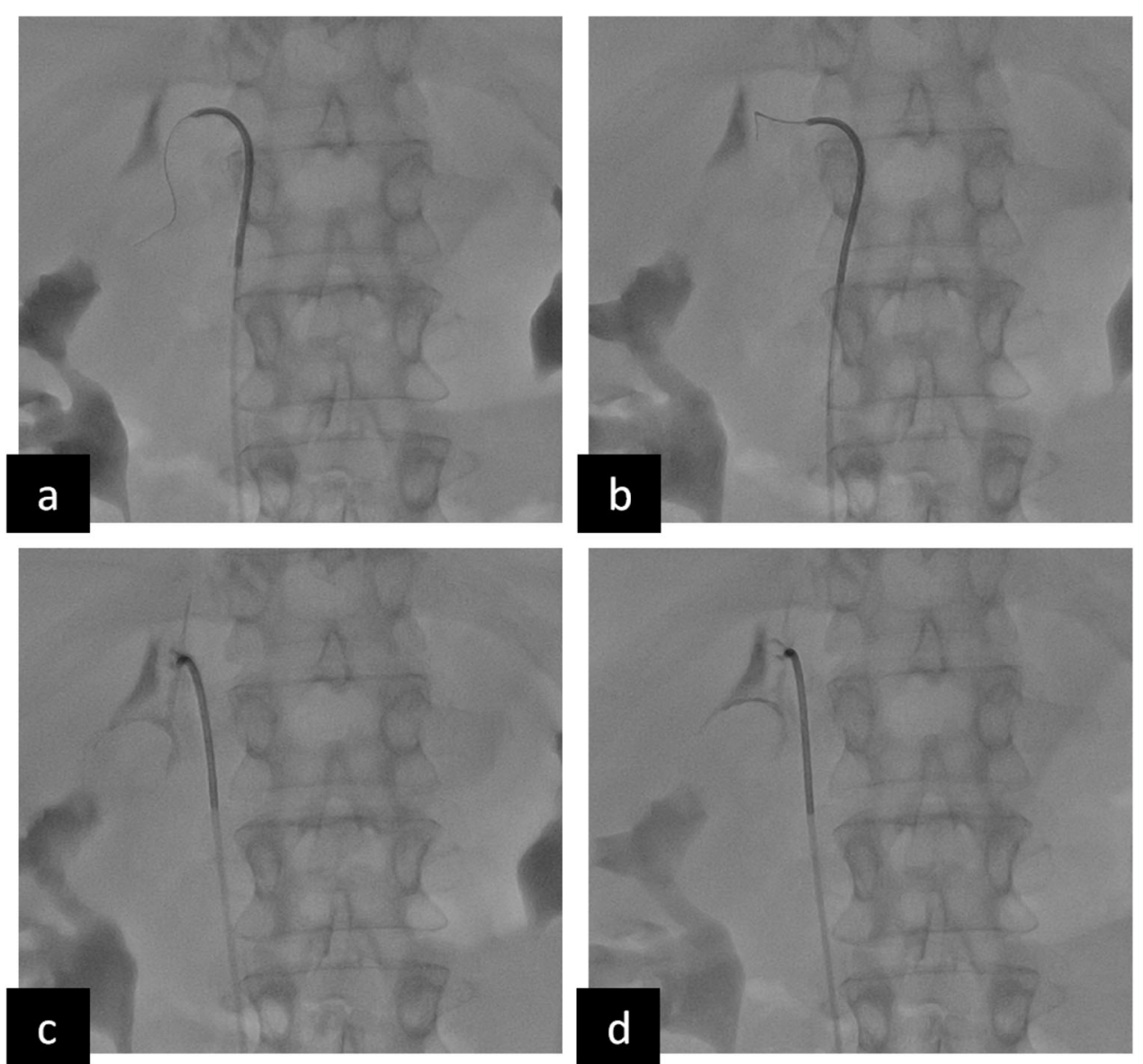

la diagnosi può essere confermata con TC o RM. La terapia, in ogni caso, è generalmente conservativa ed è rara la comparsa di un danno funzionale surrenalico a lungo termine [13].

Da uno studio multicentrico che ha coinvolto 24 centri di riferimento in tutto il mondo, è emerso come l'incidenza di rottura della vena surrenalica sia dello $0,6 \%$ e risulti inversamente proporzionale al numero di AVS eseguiti dal singolo radiologo e nel singolo centro [9].

L'AVS è quindi una procedura con un basso tasso di complicanze se eseguita da un radiologo con esperienza consolidata. Il rischio di rottura della vena surrenalica può, inoltre, essere minimizzato utilizzando la minor quantità di mezzo di contrasto possibile per verificare la posizione della punta del catetere.

\section{Confronto con le metodiche di imaging tradizionale}

Sebbene l'esecuzione di una TC surrenalica sia raccomandata in tutti i pazienti con diagnosi confermata di PA, tale indagine non può essere considerata accurata per distinguere le forme di PA unilaterali da quelle bilaterali. Ciò è dovuto alle intrinseche limitazioni della metodica nella capacità di risoluzione spaziale, che non consentono di identificare APA di dimensioni $<1 \mathrm{~cm}$ o la presenza di UAH, e alla mancanza di informazioni funzionali, non potendo differenziare gli APA dagli adenomi non funzionanti [14].

In letteratura è stata infatti riportata una discordanza tra imaging e AVS in oltre un terzo dei pazienti, con un esito post-chirurgico migliore in termini di cura dell'ipertensione arteriosa nei pazienti in cui l'indicazione alla surrenectomia è basata sui dati dell'AVS rispetto a quelli in cui le scelte terapeutiche sono basate sull'imaging [15].

L'AVS dovrebbe essere pertanto eseguito, con poche eccezioni, in tutti i pazienti con diagnosi confermata di PA che siano candidabili ad un eventuale intervento chirurgico di surrenectomia $[3,7]$.

\section{Situazioni cliniche in cui l'AVS può essere evitato}

Secondo le LG ES 2016, in pazienti di età < 35 anni, con PA florido (aldosterone plasmatico $>30 \mathrm{ng} / \mathrm{dL}$ e ipokaliemia spontanea) ed evidenza all'imaging di lesione surrenalica unilaterale $>1 \mathrm{~cm}$ compatibile con un adenoma corticale, l'AVS potrebbe essere evitato e questi pazienti inviati 
Tabella 1 Principali indici proposti per l'interpretazione dell'AVS. AVS, prelievo venoso selettivo surrenalico; $V S$, vena surrenalica; $V P$, vena periferica

\begin{tabular}{|c|c|c|c|}
\hline Indice & Formula & Interpretazione & Cut-off \\
\hline Indice di selettività (SI) & $\frac{\text { cortisolo }_{V S}}{\text { cortisolo }_{V P}}$ & $\begin{array}{l}\text { Selettività del prelievo in } \\
\text { vena surrenalica }\end{array}$ & $\begin{array}{l}\text { SI }>3 \text { per AVS basale } \\
\text { SI }>5 \text { per AVS stimolato con ACTH }\end{array}$ \\
\hline $\begin{array}{l}\text { Indice di } \\
\text { lateralizzazione (LI) }\end{array}$ & $\frac{\text { aldosterone } V_{V S} \text { dominante } / \text { cortisolo }_{V S} \text { dominante }}{\text { aldosterone } V \text { Von dominante } / \text { cortisolo }}$ & $\begin{array}{l}\text { Lateralizzazione della } \\
\text { secrezione di aldosterone }\end{array}$ & $\begin{array}{l}\mathrm{LI}>4 \text { indicativo di forma unilaterale } \\
\mathrm{LI}<3 \text { indicativo di forma bilaterale } \\
3<\mathrm{LI}<4 \text { zona grigia }\end{array}$ \\
\hline $\begin{array}{l}\text { Indice di soppressione } \\
\text { controlaterale }(\mathrm{CR})\end{array}$ & $\frac{\text { aldosterone }_{V S} \text { non dominante } / \text { cortisolo }_{V S} \text { non dominante }}{\text { aldosterone }_{V P} / \text { cortisolo }_{V P}}$ & $\begin{array}{l}\text { Soppressione della } \\
\text { secrezione di aldosterone nel } \\
\text { surrene non dominante }\end{array}$ & $\begin{array}{l}\mathrm{CR}<1 \text { predittivo di miglior outcome } \\
\text { postoperatorio nei pazienti con } \mathrm{LI} \text { in } \\
\text { zona grigia }\end{array}$ \\
\hline
\end{tabular}

direttamente all'intervento chirurgico [3]. Alcuni autori, tuttavia, diffidano da tale approccio poiché, seppur l'incidenza di masse surrenaliche non funzionanti sia bassa al di sotto dei 35 anni, la presenza di un micro-APA o di una UAH controlaterale alla massa surrenalica non può essere esclusa sulla base delle sole immagini radiologiche. Pertanto, anche in questa tipologia di pazienti, il rischio, seppur basso, di rimuovere erroneamente un surrene sano, non sembrerebbe giustificare la mancata esecuzione dell'AVS, alla luce della superiorità diagnostica e del basso tasso di complicanze in mani esperte [11].

Altre situazioni cliniche in cui l'AVS non è indicato sono la presenza documentata di FH di tipo I o di tipo III, nei quali la terapia di scelta è medica, e la presenza di un sospetto ACC poiché, in questo caso, la chirurgia sarebbe mandatoria indipendentemente dal risultato dell'AVS [10].

\section{Interpretazione dei risultati}

\section{Valutazione della selettività}

La conferma di una corretta cateterizzazione di entrambe le vene surrenaliche è un prerequisito fondamentale per l'interpretazione dei risultati dell'AVS [10]. A tale scopo, per ciascuna vena surrenalica deve essere calcolato l'indice di selettività (Selectivity Index, SI), definito come il rapporto tra il cortisolo misurato a livello della vena surrenalica e il cortisolo misurato in VCI.

Sebbene nei diversi studi presenti in letteratura siano stati proposti vari cut-off per identificare l'avvenuta cateterizzazione delle vene surrenaliche in corso di AVS [10], viene generalmente considerato accettabile un SI $\geq 2$ in condizioni basali e un SI $\geq 3$ in presenza di stimolo con cosintropina [9]; molti autori ritengono tuttavia preferibile un SI $\geq 3$ in condizioni basali e un SI $\geq 5$ in presenza di stimolo (Tabella 1) [11]. L'utilizzo di un SI troppo permissivo potrebbe compromettere l'accuratezza diagnostica dell'AVS, mentre l'uso di cut-off troppo restrittivi potrebbe portare all'esclusione di AVS adeguatamente eseguiti.

\section{Valutazione della lateralizzazione}

L'indice di lateralizzazione (Lateralization Index, LI) è il principale parametro utilizzato ai fini della diagnosi di sottotipo, per distinguere le forme di ipersecrezione di aldosterone unilaterali da quelle bilaterali.

Il LI viene calcolato come il rapporto aldosterone/ cortisolo (A/C) nella vena surrenalica dominante diviso per il rapporto A/C nella vena surrenalica non dominante. La correzione per la concentrazione di cortisolo è necessaria per minimizzare l'effetto di diluizione da parte di sangue proveniente a sinistra dalla vena frenica inferiore e a destra dalle vene epatiche accessorie [3].

Anche in questo caso, non esiste un consenso sul cut-off da utilizzare per definire la lateralizzazione; nella maggioranza dei centri, in accordo con le LG, un LI > 4 viene considerato come indicativo di secrezione unilaterale, mentre un LI $<3$ come suggestivo di secrezione bilaterale. Nei pazienti con indice di lateralizzazione compreso tra 3 e 4 , i dati dell'AVS dovrebbero essere integrati e interpretati in associazione con le caratteristiche cliniche e le immagini radiologiche, eventualmente ripetendo l'AVS qualora necessario (Tabella 1) $[3,7]$.

Alcuni autori suggeriscono che, negli AVS non stimolati, la lateralizzazione della secrezione possa essere stabilita anche sulla base di cut-off più bassi, considerando come indicativo di secrezione unilaterale un LI $>2$ [10]; tale indicazione non risulta tuttavia univocamente condivisa e deve essere, pertanto, interpretata con cautela [14].

Accanto al LI, alcuni autori hanno infine proposto, come ulteriore parametro utile ai fini della diagnosi di lateralizzazione, l'uso dell'indice di soppressione controlaterale (CR), definito come il rapporto tra l'aldosterone corretto per il cortisolo nella vena surrenalica non dominante e l'aldosterone corretto per il cortisolo in una vena periferica, risultando indicativo di soppressione controlaterale qualora sia $<1$. Alcuni studi, infatti, hanno dimostrato come, nei pazienti con un LI in zona grigia (ovvero compreso fra 3 e $4 \mathrm{o}$, con maggior cautela, tra 2 e 3), il CR possa costituire un utile stru- 
mento per guidare la successiva strategia terapeutica, essendo associato a un migliore esito postoperatorio in termini di guarigione clinica e biochimica di PA [14, 16].

\section{Problemi aperti}

- I dati disponibili non risultano sufficienti per dirimere il dubbio circa la differente accuratezza delle tecniche di AVS con prelievo sequenziale o simultaneo.

- È stato ipotizzato che la somministrazione di cosintropina possa stimolare la secrezione di aldosterone da parte del surrene controlaterale a un APA, determinando una riduzione del LI [11]. Tuttavia, ad oggi non vi sono sufficienti evidenze sulla superiorità dell'AVS basale rispetto all'AVS stimolato in termini di esito post-surrenectomia.

- Negli ultimi anni alcuni studi $[17,18]$ non hanno dimostrato una netta superiorità, in termini di outcome, della surrenectomia AVS-guidata rispetto all'intervento eseguito solo sulla base dell'imaging in TC. Tali studi sono stati fortemente criticati per il disegno e, pertanto, si ritiene che non vi siano prove sufficienti a confermarne i risultati.

- L'impatto clinico della presenza o meno della soppressione della secrezione di aldosterone nel surrene controlaterale a una forma unilaterale di PA risulta controversa dagli studi disponibili in letteratura [19, 20].

- Per la valutazione della selettività dell'AVS sono stati proposti parametri alternativi al cortisolo (in particolare catecolamine, metanefrine, androstenedione e $17-\alpha$ idrossiprogesterone), che potrebbero essere di particolare utilità in presenza di una co-secrezione aldosteronecortisolo [7].

\section{Conclusioni}

Il PA è la causa più frequente di ipertensione arteriosa secondaria e si associa a un significativo aumento del rischio cardiovascolare; pertanto, una corretta diagnosi e un adeguato trattamento risultano fondamentali.

L'AVS rimane ad oggi il gold standard nella diagnosi di sottotipo. In mani esperte, risulta caratterizzato da un buon tasso di successo e da un basso rischio di complicanze. Con poche eccezioni, risulta pertanto mandatoria la sua esecuzione presso un centro di riferimento, al fine di selezionare al meglio i pazienti da indirizzare alla chirurgia.

Funding Note Open Access funding provided by Università degli Studi di Torino.

Conflitto di interesse Gli autori Martina Bollati, Fabio Bioletto, Chiara Lopez, Mirko Parasiliti-Caprino, Ezio Ghigo e Mauro Maccario dichiarano di non avere conflitti di interesse.
Consenso informato Lo studio presentato in questo articolo non ha richiesto sperimentazione umana.

Studi sugli animali Gli autori di questo articolo non hanno eseguito studi sugli animali.

Nota della casa editrice Springer Nature rimane neutrale in riguardo alle rivendicazioni giurisdizionali nelle mappe pubblicate e nelle affiliazioni istituzionali.

Open Access This article is licensed under a Creative Commons Attribution 4.0 International License, which permits use, sharing, adaptation, distribution and reproduction in any medium or format, as long as you give appropriate credit to the original author(s) and the source, provide a link to the Creative Commons licence, and indicate if changes were made. The images or other third party material in this article are included in the article's Creative Commons licence, unless indicated otherwise in a credit line to the material. If material is not included in the article's Creative Commons licence and your intended use is not permitted by statutory regulation or exceeds the permitted use, you will need to obtain permission directly from the copyright holder. To view a copy of this licence, visit http://creativecommons.org/licenses/by/4.0/.

\section{Bibliografia}

1. Rossi GP, Bernini G, Caliumi C et al (2006) A prospective study of the prevalence of primary aldosteronism in 1,125 hypertensive patients. J Am Coll Cardiol 48:2293-2300

2. Parasiliti-Caprino M, Lopez C, Prencipe N et al (2020) Prevalence of primary aldosteronism and association with cardiovascular complications in patients with resistant and refractory hypertension. J Hypertens 38(9): 1841-1848

3. Funder JW, Carey RM, Mantero F et al (2016) The management of primary aldosteronism: case detection, diagnosis, and treatment: an Endocrine Society clinical practice guideline. J Clin Endocrinol Metab 101:1889-1916

4. Monticone S, D'Ascenzo F, Moretti C et al (2018) Cardiovascular events and target organ damage in primary aldosteronism compared with essential hypertension: a systematic review and meta-analysis. Lancet Diabetes Endocrinol 6:41-50

5. Rossi GP, Cesari M, Cuspidi C et al (2013) Long-term control of arterial hypertension and regression of left ventricular hypertrophy with treatment of primary aldosteronism. Hypertension 62:62-69

6. Reincke M, Fischer E, Gerum S et al (2012) Observational study mortality in treated primary aldosteronism: the German Conn's registry. Hypertension 60:618-624

7. Rossi GP, Bisogni V, Bacca AV et al (2020) The 2020 Italian Society of Arterial Hypertension (SIIA) practical guidelines for the management of primary aldosteronism. Int $\mathbf{J}$ Cardiol Hypertens 5:100029

8. Williams TA, Lenders JW, Mulatero P et al (2017) Outcomes after adrenalectomy for unilateral primary aldosteronism: an international consensus on outcome measures and analysis of remission rates in an international cohort. Lancet Diabetes Endocrinol 5:689-699

9. Rossi GP, Barisa M, Allolio B et al (2012) The adrenal vein sampling International study (AVIS) for identifying the major subtypes of primary aldosteronism. J Clin Endocrinol Metab 97:1606-1614

10. Rossi GP, Auchus RJ, Brown M et al (2014) An expert consensus statement on use of adrenal vein sampling for the subtyping of primary aldosteronism. Hypertension 63:151-160

11. Monticone S, Viola A, Rossato D et al (2015) Adrenal vein sampling in primary aldosteronism: towards a standardised protocol. Lancet Diabetes Endocrinol 3(4):296-303 
12. Mengozzi G, Rossato D, Bertello C et al (2007) Rapid cortisol assay during adrenal vein sampling in patients with primary aldosteronism. Clin Chem 53:1968-1971

13. Monticone S, Satoh F, Dietz AS et al (2016) Clinical management and outcomes of adrenal hemorrhage following adrenal vein sampling in primary aldosteronism. Hypertension 67:146-152

14. Buffolo F, Monticone S, Williams T et al (2017) Subtype diagnosis of primary aldosteronism: is adrenal vein sampling always necessary? Int J Mol Sci 18:848

15. Rossi GP, Rossitto G, Amar L et al (2019) Clinical outcomes of 1625 patients with primary aldosteronism subtyped with adrenal vein sampling. Hypertension 74:800-808

16. Wolley MJ, Gordon RD, Ahmed AH et al (2015) Does contralateral suppression at adrenal venous sampling predict outcome following unilateral adrenalectomy for primary aldosteronism? A retrospective study. J Clin Endocrinol Metab 100:1477-1484
17. Dekkers T, Prejbisz A, Kool LJ et al (2016) Adrenal vein sampling versus CT scan to determine treatment in primary aldosteronism: an outcome-based randomised diagnostic trial. Lancet Diabetes Endocrinol 4:739-746

18. Williams TA, Burrello J, Sechi LA et al (2018) Computed tomography and adrenal venous sampling in the diagnosis of unilateral primary aldosteronism. Hypertension 72:641-649

19. Monticone S, Satoh F, Viola A et al (2014) Aldosterone suppression on contralateral adrenal during adrenal vein sampling does not predict blood pressure response after adrenalectomy. J Clin Endocrinol Metab 99:4158-4166

20. Tagawa M, Ghosn M, Wachtel H et al (2017) Lateralization index but not contralateral suppression at adrenal vein sampling predicts improvement in blood pressure after adrenalectomy for primary aldosteronism. J Hum Hypertens 31:444-449 